\title{
A Mathematical Theorem on the Onset of Stationary Convection in Couple-Stress Fluid
}

\author{
A. S. Banyal \\ Department of Mathematics, NSCBM, Govt. College Hamirpur, (HP,) 177005, India
}

Email:ajaibbanyal@rediffmail.com

(Received April 1, 2011; accepted August 6, 2011)

\begin{abstract}
The thermal instability of a couple-stress fluid heated from below is investigated. Following the linearized stability theory and normal mode analysis, the paper mathematically establishes that the onset of instability at marginal state, cannot manifest itself as stationary convection, if the thermal Rayleigh number R and the couple-stress parameter F, satisfy the inequality $R \leq \frac{\left(2+\sqrt{1+3 \pi^{2} F}\right)\left(3 \pi^{2} F+\sqrt{1+3 \pi^{2} F}-1\right)^{3}}{27 F^{2}\left(-1+\sqrt{\left(1+3 \pi^{2} F\right)}\right)}$, and when the couple-stress parameter $\mathrm{F}$ is infinitesimally small, $R \leq \frac{27 \pi^{4}}{4}\left\{1+\frac{\pi^{2}}{2} F\right\}$, the result which also clearly mathematically established the stabilizing character of the couple-stress.
\end{abstract}

Keywords: Thermal convection; Couple-stress fluid; Rayleigh number.

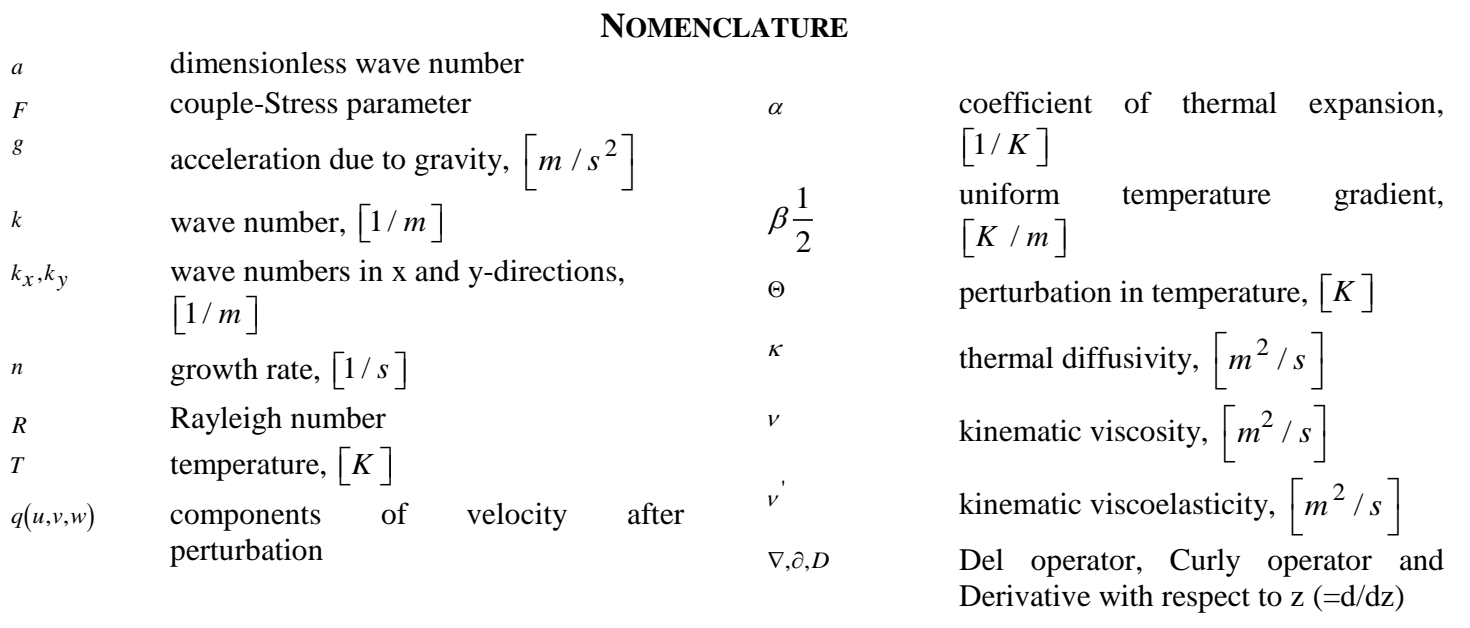

\section{INTRODUCTION}

Right from the conceptualizations of turbulence, instability of fluid flows is being regarded at its root. The thermal instability of a fluid layer with maintained adverse temperature gradient by heating the underside plays an important role in Geophysics, interiors of the Earth, Oceanography and Atmospheric Physics etc. A detailed account of the theoretical and experimental study of the onset of Bénard Convection in Newtonian fluids, under varying assumptions of hydrodynamics and hydromagnetics, has been given by Chandrasekhar

(1981). The use of Boussinesq approximation has been made throughout, which states that the density changes are disregarded in all other terms in the equation of motion except the external force term. Sharma (1976) has considered the effect of suspended particles on the onset of Bénard convection in hydromagnetics. The fluid has been considered to be Newtonian in all above studies. Chandra (1938) observed that in an air layer, convection occurred at much lower gradients than predicted if the layer depth was less than $7 \mathrm{~mm}$ and called this motion "columnar instability". However for 
a layer deeper than $10 \mathrm{~mm}$, Bénard-type cellular convection was observed. Thus there is a contradiction between the theory and experiment. Scanlon and Segel (1973) have considered the effect of suspended particles on the onset of Bénard convection and found that the critical Rayleigh number was reduced solely because the heat capacity of the pure fluid was supplemented by that of the particles.

With the growing importance of non-Newtonian fluids in modern technology and industries, the investigations on such fluids are desirable. Stokes (1966) proposed and postulated the theory of couple-stress fluid. One of the applications of couple-stress fluid is its use to the study of the mechanism of lubrication of synovial joints, which has become the object of scientific research. A human joint is a dynamically loaded bearing which has articular cartilage as the bearing and synovial fluid as lubricant. When fluid film is generated, squeeze film action is capable of providing considerable protection to the cartilage surface. The shoulder, knee, hip and ankle joints are the loadedbearing synovial joints of human body and these joints have low-friction coefficient and negligible wear. Normal synovial fluid is clear or yellowish and is a viscous, non-Newtonian fluid. According to the theory of Stokes (1966), couple-stresses are found to appear in noticeable magnitude in fluids very large molecules. Since the long chain hylauronic acid molecules are found as additives in synovial fluid. Walicki and Walicka (1999) modeled synovial fluid as couple-stress fluid in human joints. Sharma and Sharma (2001) have studied the couple-stress fluid heated from below in porous medium. The use of magnetic field is being made for the clinical purposes in detection and cure of certain diseases with the help of magnetic field devices. Sharma and Thakur (2000) have studied the thermal convection in couple-stress fluid in porous medium in hydromagnetics.

Sharma and Sharma (2004) have studied the effect of suspended particles on couple-stress fluid heated from below in the presence of rotation and magnetic field and found that rotation has a stabilizing effect while dust particles have a destabilizing effect on the system. Sunil et al. (2004) have studied the effect of suspended particles on couple-stress fluid heated and soluted from below in porous medium and found that suspended particles have stabilizing effect on the system. Kumar and Kumar (2011) have studied the combined effect of dust particles, magnetic field and rotation on couplestress fluid heated from below and for the case of stationary convection, found that dust particles have destabilizing effect on the system, where as the rotation is found to have stabilizing effect on the system, however couple-stress and magnetic field are found to have both stabilizing and destabilizing effects under certain conditions. Shivakumara et al. (2011) have studied the effect of non-uniform temperature gradients on the onset of convection in a couple-stress fluid saturated porous medium.

Keeping in mind the importance of non-Newtonian fluids, the present paper is an attempt to characterize the onset of instability at marginal state as stationary convection analytically, in a layer of incompressible couple-stress fluid heated from below in the presence of suspended particles.

\section{Formulation Of The Problem And Perturbation EQuations}

Considered an infinite, horizontal, incompressible couple-stress fluid layer, of thickness $d$, heated from below so that, the temperature and density at the bottom surface $\mathrm{z}=0$ are, $\rho_{0}$ respectively and at the lower surface $\mathrm{z}=\mathrm{d}$ are $T_{d}, \rho_{d}$ and that a uniform adverse temperature gradient $\beta\left(=\left|\frac{d T}{d z}\right|\right)$ is maintained. Let $\rho, \mathrm{p}$, $\mathrm{T}$ and $\vec{q}(u, v, w)$ denote respectively the density, pressure, temperature and velocity of the fluid respectively. Then the momentum balance, mass balance equations of the couple-stress fluid (Stokes 1966, Chandrasekhar 1981 and Scanlon and Segel 1973) are

$\frac{\partial \vec{q}}{\partial t}+(\vec{q} \cdot \nabla) \vec{q}=-\frac{1}{\rho_{0}} \nabla p+\vec{g}\left(1+\frac{\delta \rho}{\rho_{0}}\right)+\left(v-\frac{\mu^{\prime}}{\rho_{0}} \nabla^{2}\right) \nabla^{2} \vec{q}$

$\nabla \cdot \vec{q}=0$

The equation of state is

$\rho=\rho_{0}\left[1-\alpha\left(T-T_{0}\right)\right]$

Where the suffix zero refer to the values at the reference level $z=0$. Here $\vec{g}(0,0,-g)$ is acceleration due to gravity and $\bar{x}=(x, y, z)$.

Let $c_{v}$ denote the heat capacity of the fluid at constant volume, assuming that the fluid is in thermal equilibrium, the equation of heat conduction gives

$\rho_{0} c_{v}\left(\frac{\partial}{\partial t}+\vec{q} \cdot \nabla\right) T=\vec{q} \nabla^{2} T$

or

$\frac{\partial T}{\partial t}+(\vec{q} \cdot \nabla) T=\kappa \nabla^{2} T$

The kinematic viscosity $\nu$, couple-stress viscosity $\mu^{\prime}$, thermal diffusivity $\kappa=q / \rho_{0} c_{v}$, and coefficient of thermal expansion $\alpha$ are all assumed to be constants.

The initial state of the system is taken to be a quiescent layer (no settling). The basic motionless solution is given by

$\vec{q}=(0,0,0), T=T_{0}-\beta z, \rho=\rho_{0}(1+\alpha \beta z)$

Assume small perturbations around the basic solution and let $\delta \rho, \delta p, \theta$ and $q(u, v, w)$ denote respectively 
the perturbations in density, pressure $\mathrm{p}$, temperature $\mathrm{T}$ and couple-stress fluid velocity $(0,0,0)$. The change in density $\delta \rho$ caused mainly by the perturbation $\theta$ in temperature is given by:

$$
\delta \rho=-\alpha \rho_{0} \theta
$$

Then the linearized perturbation equations of the couple stress fluid become

$$
\begin{aligned}
& \frac{\partial \vec{q}}{\partial t}=-\frac{1}{\rho_{0}} \nabla \delta p-\vec{g} \alpha \theta+\left(v-\frac{\mu^{\prime}}{\rho_{0}} \nabla^{2}\right) \nabla^{2} \vec{q} \\
& \nabla \cdot \vec{q}=0 \\
& \frac{\partial \theta}{\partial t}=\beta w+\kappa \nabla^{2} \theta
\end{aligned}
$$

\section{NORMAL MODE ANALYSIS}

Analyzing the disturbances into normal modes, we assume that the Perturbation quantities are of the form

$$
[w, \theta]=[W(z), \Theta(z)] \operatorname{Exp}\left(i k_{x} x+i k_{y} y+n t\right)
$$

Where $k_{x}, k_{y}$ are the wave numbers along the $\mathrm{x}$ and $\mathrm{y}$ directions respectively $k=\left(k_{x}^{2}+k_{y}^{2}\right)^{\frac{1}{2}}, \quad$ is the resultant wave number and $\mathrm{n}$ is the growth rate which is, in general, a complex constant.

Using Eq. (10), Eq. (7) and Eq. (9), on using Eq. (8) and, in non-dimensional form, become

$\left(D^{2}-a^{2}\right)\left[\sigma+F\left(D^{2}-a^{2}\right)^{2}-\left(D^{2}-a^{2}\right)\right] W$

$=-\frac{g \alpha d^{2} a^{2} \Theta}{v}$

$\left(D^{2}-a^{2}-p_{1} \sigma\right) \Theta=-\frac{\beta d^{2}}{\kappa} W$

where $a=k d, \sigma=\frac{n d^{2}}{v}, p_{1}=\frac{v}{\kappa}, F=\frac{\mu}{\rho_{0} d^{2} v}, D=\frac{d}{d z}$ and dropping $(\oplus)$ for convenience. Here $p_{1}=\frac{v}{\kappa}$, is the thermal prandtl number and $\mathrm{F}$ is the couple-stress parameter.

Substituting $W=W_{\oplus}$ and $\Theta=\frac{\beta d^{2}}{\kappa} \Theta_{\oplus}$ in Eq.

and Eq. (12) and dropping $(\oplus)$ for convenience, in non-dimensional form becomes

$$
\begin{aligned}
& \left(D^{2}-a^{2}\right)\left[\sigma+F\left(D^{2}-a^{2}\right)^{2}-\left(D^{2}-a^{2}\right)\right] W=-R a^{2} \Theta \\
& \left(D^{2}-a^{2}-p_{1} \sigma\right) \Theta=-W
\end{aligned}
$$

where $R=\frac{g \alpha \beta d^{4}}{\kappa v}$, is the thermal Rayleigh number.

Since both the boundaries are maintained at constant temperature, the perturbations in the temperature are zero at the boundaries. The case of two free boundaries is little artificial but it is most appropriate for Stellar atmospheres and enables us to find analytical solutions and to make some qualitative and quantitative conclusions. The appropriate boundary conditions with respect to which Eq. (13) and Eq. (14) must be solved are

$\mathrm{W}=0, \quad \Theta=0$ at $\mathrm{z}=0$ and $\mathrm{z}=1$

and the constitutive equations of the couple-stress fluid are $\tau_{i j}=\left(2 \mu-2 \mu^{\prime} \nabla^{2}\right) e_{i j}$ and $e_{i j}=\frac{1}{2}\left(\frac{\partial v_{i}}{\partial x_{j}}+\frac{\partial v_{j}}{\partial x_{i}}\right)$

The conditions on a free surface are

$$
\begin{gathered}
\tau_{x z}=\left(\mu-\mu^{\prime} \nabla^{2}\right)\left(\frac{\partial u}{\partial z}+\frac{\partial w}{\partial x}\right)=0 \\
\tau_{y z}=\left(\mu-\mu^{\prime} \nabla^{2}\right)\left(\frac{\partial v}{\partial z}+\frac{\partial w}{\partial y}\right)=0
\end{gathered}
$$

From the equation of continuity Eq. (8) differentiating with respect to $\mathrm{z}$, we conclude that

$$
\left[\mu-\mu^{\prime}\left(\frac{\partial^{2}}{\partial x^{2}}+\frac{\partial^{2}}{\partial y^{2}}+\frac{\partial^{2}}{\partial z^{2}}\right)\right] \frac{\partial^{2} w}{\partial z^{2}}=0
$$

which implies that

$$
\frac{\partial^{2} w}{\partial z^{2}}=0, \frac{\partial^{4} w}{\partial z^{4}}=0, \text { at } \mathrm{z}=0 \text { and } \mathrm{z}=\mathrm{d}
$$

Using Eq. (10), the boundary conditions Eq. (18) in non-dimensional form transform to

$D^{2} W=D^{4} W=0$ at $\mathrm{z}=0$ and $\mathrm{z}=1$

We prove the following theorem.

Theorem: If R>0, F> 0 and $\sigma=0$ then the necessary condition for the existence of non-trivial solution $(W, \Theta)$ of Eqs. (13) and (14) together with boundary conditions Eqs. (15) and (19) is that

$$
R>\frac{\left(2+\sqrt{1+3 \pi^{2} F}\right)\left(3 \pi^{2} F+\sqrt{1+3 \pi^{2} F}-1\right)^{3}}{27 F^{2}\left(-1+\sqrt{\left(1+3 \pi^{2} F\right)}\right)}
$$

further, when the couple-stress parameter $\mathrm{F}$ is infinitesimally small, then $\mathrm{R}>\frac{27 \pi^{4}}{4}\left\{1+\frac{\pi^{2}}{2} F\right\}$ 
Proof: When the instability sets in stationary convection and the 'exchange principle' is valid, the neutral or marginal state will be characterized by $\sigma=0$ and hence the relevant governing Eqs. (13) and (14) reduces to

$$
\begin{aligned}
& \left(D^{2}-a^{2}\right)\left[F\left(D^{2}-a^{2}\right)^{2}-\left(D^{2}-a^{2}\right)\right] W \\
& =-R a^{2} \Theta \\
& \left(D^{2}-a^{2}\right) \Theta=-W
\end{aligned}
$$

together with the relevant boundary conditions,

$W=0=\Theta=D^{2} W=D^{4} W$

on both the horizontal boundaries at $\mathrm{z}=0$ and $\mathrm{z}=1$

Multiplying Eq. (20) by $W^{*}$ (the complex conjugate of $\mathrm{W})$ throughout and integrating the resulting equation over the vertical range of $\mathrm{z}$, we get

$$
\begin{aligned}
& F \int_{0}^{1} W^{*}\left(D^{2}-a^{2}\right)^{3} W d z-\int_{0}^{1} W^{*}\left(D^{2}-a^{2}\right)^{2} W d z \\
& =-R a^{2} \int_{0}^{1} W^{*} \Theta d z
\end{aligned}
$$

Taking complex conjugate on both sides of Eq. (21), we get

$$
\left(D^{2}-a^{2}\right) \Theta^{*}=-W^{*}
$$

Substituting for $W^{*}$ in the right hand side of Eq. (23), we get

$$
\begin{aligned}
& F \int_{0}^{1} W^{*}\left(D^{2}-a^{2}\right)^{3} W d z-\int_{0}^{1} W^{*}\left(D^{2}-a^{2}\right)^{2} W d z \\
& =R a^{2} \int_{0}^{1} \Theta^{*}\left(D^{2}-a^{2}\right) \Theta d z
\end{aligned}
$$

Integrating the terms on both sides of Eq. (25) for an appropriate number of times by making use of the appropriate boundary conditions Eq. (22), we get

$$
\begin{aligned}
& F \int_{0}^{1}\left\{\left|D^{3} W\right|^{2}+3 a^{2}\left|D^{2} W\right|^{2}+3 a^{4}|D W|^{2}+a^{6}|W|^{2}\right\} d z \\
& +\int_{0}^{1}\left\{\left|D^{2} W\right|^{2}+2 a^{2}|W|^{2}+a^{4}|W|^{2}\right\} d z \\
& =R a^{2} \int_{0}^{1}\left\{|D \Theta|^{2}+a^{2}|\Theta|^{2}\right\} d z
\end{aligned}
$$

We first note that since $W$ and $\Theta$ satisfy $W(0)=0=W(1)$ and $\Theta(0)=0=\Theta(1)$ in addition to satisfying to governing equations and hence we have from the Rayleigh-Ritz (1973) inequality

$$
\int_{0}^{1}|D W|^{2} d z \geq \pi_{0}^{2} \int_{0}^{1}|W|^{2} d z
$$

$\int_{0}^{1}|D \Theta|^{2} d z \geq \pi^{2} \int_{0}^{1}|\Theta|^{2} d z$

Further, for $W(0)=0=W(1)$, Banerjee et al. (1992) have shown that

$\int_{0}^{1}\left|D^{2} W\right|^{2} d z \geq \pi_{0}^{4} \int_{0}^{1}|W|^{2} d z$

Further, multiplying Eq. (21) by $\Theta^{*}$ (the complex conjugate of $\Theta$ ), integrating by parts each term of the resulting equation on the right hand side for an appropriate number of times and making use of boundary condition on $\Theta$ namely $\Theta(0)=0=\Theta(1)$, it follows that

$\int_{0}^{1}\left\{|D \Theta|^{2}+a^{2}|\Theta|^{2}\right\} d z=$ Real part

$\int_{0}^{1}\left\{|D \Theta|^{2}+a^{2}|\Theta|^{2}\right\} d z=$ Real part of $\left\{\begin{array}{l}1 \\ \int \\ 0\end{array} \Theta^{*} W d z\right\}$

$\leq\left|\int_{0}^{1} \Theta^{*} W d z\right|$

$\leq \int_{0}^{1}\left|\Theta^{*} W\right| d z$

$\leq \int_{0}^{1}\left|\Theta^{*}\right||W| d z$

1

$\leq \int|\Theta||W| d z$

$\leq\left\{\int_{0}^{1}|\Theta|^{2} d z\right\}^{\frac{1}{2}}\left\{\int|W|^{2} d z\right\}^{\frac{1}{2}}$

(Utilizing Cauchy - Schwartz - inequality)

So that using inequality Eq. (28), we obtained from above inequality

$\left(\pi^{2}+a^{2}\right)\left\{\int|\Theta|^{2} d z\right\}^{\frac{1}{2}} \leq\left\{\int|W|^{2} d z\right\}^{\frac{1}{2}}$

And hence inequality Eqs. (30) and (31), gives

$\int_{0}^{1}\left\{|D \Theta|^{2}+a^{2}|\Theta|^{2}\right\} d z \leq \frac{1}{\left(\pi^{2}+a^{2}\right)} \int_{0}^{1}|W|^{2} d z$

Now, if R >0, utilizing the inequalities Eqs. (27), (29), and Eq. (32), the Eq. (26) gives

$I_{1}+\left[\left\{a^{2}\left(\pi^{2}+a^{2}\right)^{2} F+\left(\pi^{2}+a^{2}\right)^{2}\right\}-\frac{R a^{2}}{\left(\pi^{2}+a^{2}\right)}\right] \int_{0}^{1}|W|^{2} d z<1$ 
Where $I_{1}=\int_{0}^{1}\left\{\left|D^{3} W\right|^{2}+2 a^{2}\left|D^{2} W\right|^{2}+a^{4}|D W|^{2}\right\} d z$,

positive definite. and therefore, we must have

$R>\frac{\left(\pi^{2}+a^{2}\right)^{3}}{a^{2}}\left\{1+a^{2} F\right\}$

and thus we necessarily have

$R>\frac{\left(2+\sqrt{1+3 \pi^{2} F}\right)\left(3 \pi^{2} F+\sqrt{1+3 \pi^{2} F}-1\right)^{3}}{27 F^{2}\left(-1+\sqrt{\left(1+3 \pi^{2} F\right)}\right)}$

Since the minimum value of $\frac{\left(\pi^{2}+a^{2}\right)^{3}}{a^{2}}\left\{1+a^{2} F\right\}$, is

$$
\begin{aligned}
& \frac{\left(2+\sqrt{1+3 \pi^{2} F}\right)\left(3 \pi^{2} F+\sqrt{1+3 \pi^{2} F}-1\right)^{3}}{27 F^{2}\left(-1+\sqrt{\left(1+3 \pi^{2} F\right)}\right)} \\
& \text { for } a^{2}=\frac{-1+\sqrt{1+3 \pi^{2} F}}{3 F}>0
\end{aligned}
$$

Further, when the couple-stress parameter $F$ is infinitesimally small, then in the expansion of right hand side of Eq. (36), the higher powers of $F$ can be ignored, and then we have $a^{2}=\frac{\pi^{2}}{2}>0$, Eq. (34) gives

$$
R>\frac{27 \pi^{4}}{4}\left\{1+\frac{\pi^{2}}{2} F\right\}
$$

The same result Eq. (37) also follows for very-very small values of $\mathrm{F}$ and ignoring the higher powers of $\mathrm{F}$ in the expansion of right hand side of Eq. (35) and this completes the proof of the theorem.

The theorem implies from the physical point of view that the onset of instability at marginal state in a couplestress fluid heated from below, cannot manifest itself as stationary convection, if the thermal Rayleigh number $\mathrm{R}$ and the couple-stress parameter $\mathrm{F}$, satisfy the inequality $R \leq \frac{27 \pi^{4}}{4}\left\{1+\frac{\pi^{2}}{2} F\right\}$, when couple-stress parameter is infinitesimally small and the boundaries are dynamically free.

\section{CONClusions}

In this paper, the onset of instability as stationary convection of couple-stress fluid heated from below is considered and the immediate analytic conclusions of the theorem proved above, are as follows:

(a) The necessary condition for the onset of instability as stationary convection at marginal state for configuration under consideration, is that the inequality (35) is satisfied. Thus the sufficient condition for the non-existence of stationary convection at marginal state is is that $R \leq \frac{\left(2+\sqrt{1+3 \pi^{2} F}\right)\left(3 \pi^{2} F+\sqrt{1+3 \pi^{2} F}-1\right)^{3}}{27 F^{2}\left(-1+\sqrt{\left(1+3 \pi^{2} F\right)}\right)}$, for the configuration under consideration.

(b) When the couple-stress parameter $F$ is infinitesimally small, then the necessary condition for the onset of instability as stationary convection at marginal state for configuration under consideration is that the inequality (37) is satisfied. Thus the sufficient condition for the non-existence of stationary convection at marginal state is that $R \leq \frac{27 \pi^{4}}{4}\left\{1+\frac{\pi^{2}}{2} F\right\}$, for the configuration under consideration.

(c) In the inequalities (35) and (37), the thermal Rayleigh number $\mathrm{R}>0$, is directly proportional to the couple-stress parameter $\mathrm{F}\rangle 0$, which clearly mathematically established the stabilizing character of the couple-stress, for the configuration under consideration as derived by Sharma and Sharma (2004).

\section{ACKNOWLEDGMENT}

The author is highly thankful to the referees for their very constructive, valuable suggestions and useful technical comments, which led to a significant improvement of the paper.

\section{REFERENCES}

Banerjee, M.B., Gupta, J.R. and Prakash, J. (1992). On thermohaline convection of Veronis type, J. Math Anal. Appl., Vol.179, 327-334.

Chandrasekhar, S. (1981). Hydrodynamic and Hydromagnetic Stability, Dover Publication, NewYork

Chandra, K., (1938). Stability of fluids heated from below, Proc. Roy. Soc. London, vol. A164, 231

Kumar, V. and Kumar, S. (2011). On a couple-stress fluid heated from below in hydromagnetics, Appl. Appl. Math., Vol. 05(10), 1529-1542

Scanlon, J.W. and Segel, L.A. (1973). Some effects of suspended particles on the onset of Bénard convection, Phys. Fluids. Vol. 16, 1573-1578

Schultz, M.H. (1973). Spline Analysis, Prentice Hall, Englewood Cliffs, New Jersy.

Sharma, R.C., Prakash, K. and Dube, S.N. (1976).Effect of suspended particles on the onset of Bénard convection in hydromagnetics, J. Math. Anal. Appl., USA, Vol. 60 , 227-35.

Sharma, R.C. and Thakur, K. D. (2000). Couple stressfluids heated from below in hydromagnetics, Czech. J. Phys., Vol. 50, 753-58 
A. S. Banyal et al. / JAFM, Vol. 6, No. 2, pp. 191-196, 2013.

Sharma, R.C. and Sharma, M. (2004). Effect of suspended particles on couple-stress fluid heated from below in the presence of rotation and magnetic field, Indian J. pure. Appl. Math., 35(8), 973-989

Sharma, R.C. and Sharma S. (2001). On couple-stress fluid heated from below in porous medium, Indian J. Phys, Vol. 75B, 59-61.

Shivakumar, I.S., Kumar, S.S. andDevaraju, N. (2011), "Effect of non- uniform temperature gradients on the onset of convection in a couple-stress fluid saturatd porous medium", J. of Appld Fluid Mechanics, (Accepted for publications).
Stokes, V.K.(1966). Couple-stress in fluids, Phys. Fluids, Vol. 9, 1709-1715.

Sunil, Sharma, R.C. and Chandel, R.S. (2004). Effect of suspended particles on couple-stress fluid heated and soluted from below in porous medium, J. of Porous Media, 7(1) 9-18.

Walicki, E.and Walicka, A. (1999). Inertial effect in the squeeze film of couple-stress fluids in biological bearings, Int. J. Appl. Mech. Engg., Vol. 4, 363373. 